
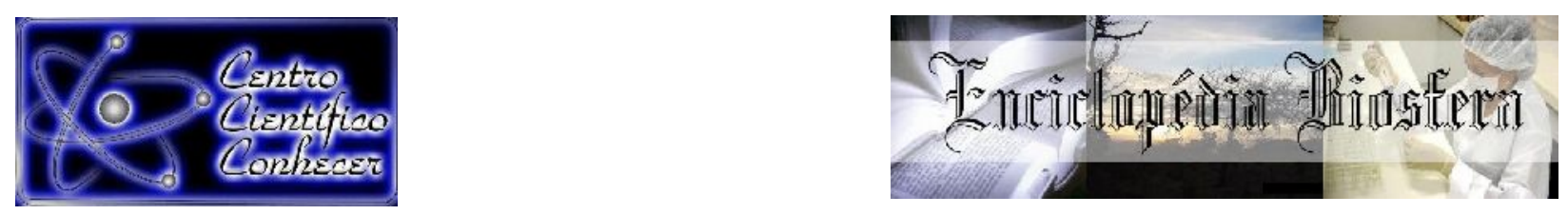

\title{
MODELAGEM DE DISTRIBUIÇÃO DE CANDEIA PARA MINAS GERAIS
}

Wander Amaral Gladson ${ }^{1}$, Múcio Magno de Melo Farnezi ${ }^{2}$, Israel Marinho Pereira ${ }^{3}$; Evandro Luiz Mendonça Machado ${ }^{4}$, Michele Aparecida Pereira da Silva ${ }^{5}$

${ }^{1}$ Doutorando no programa de pós-graduação em Ciências Florestais da Universidade Federal dos Vales do Jequitinhonha e Mucuri- UFVJM. Email: wander.amaral@yahoo.com.br

${ }^{2}$ Doutorando no programa de pós-graduação em Produção Vegetal da Universidade Federal dos Vales do Jequitinhonha e Mucuri- UFVJM.

${ }^{3,4}$ Professor adjunto no Departamento de Engenharia Florestal da Universidade

Federal dos Vales do Jequitinhonha e Mucuri - UFVJM.

${ }^{5}$ Pós-doutoranda no Departamento de Engenharia Florestal da Universidade Federal de Lavras- UFLA.

Recebido em: 22/09/2018 - Aprovado em: 23/11/2018 - Publicado em: 03/12/2018 DOI: 10.18677/EnciBio_2018B59

\begin{abstract}
RESUMO
A espécie Eremanthus erythropappus (DC). Macleish, pertencente à família Asteraceae, do grupo ecológico das pioneiras, é considerada precursora na invasão de campos. Apesar disso, pouco há na literatura sobre a distribuição das espécies desse gênero no estado de Minas Gerais. Assim, este trabalho teve por objetivo gerar modelos da distribuição potencial atual de E. erythropappus no estado de Minas Gerais, a fim de fornecer informações que servirão como estratégia para o seu uso sustentável, conservação, restauração e domesticação. Para isso, foram utilizados 23 variáveis ambientais, sendo 19 bioclimáticas (extraídas do Worldclim), e quatro variáveis topográficas do território de Minas Gerais, extraídas do modelo digital de elevação por meio do software ArcGis 10.2.2. A modelagem de distribuição da espécie E. erythropappus no estado de Minas Gerais condiz com os dados de ocorrência da espécie. Assim, as informações geradas poderão subsidiar estudos e manejos mais sustentáveis, buscando a conservação, restauração e domesticação da espécie. Além disto, o software Maxent apresentou um alto poder preditivo.
\end{abstract}

PALAVRAS-CHAVE: distribuição potencial, espécies vegetais, modelagem.

\section{CANDEIA DISTRIBUTION MODELING FOR MINAS GERAIS}

\begin{abstract}
The species Eremanthus erythropappus (DC). Macleish, belonging to the Asteraceae family, of the ecological group of the pioneers, is considered precursor in the invasion of fields. Despite this, there is little in the literature on the distribution of species of this genus in the state of Minas Gerais. Thus, this work aims to generate models of the current potential distribution of E. erythropappus in the state of Minas Gerais, in order to provide information that will serve as a strategy for its sustainable use, conservation, restoration and domestication. For this, 23 environmental variables
\end{abstract}


were used, of which 19 were bioclimatic (extracted from the Worldclim), and four topographic variables from the territory of Minas Gerais, extracted from the digital elevation model using the ArcGis 10.2.2 software. The distribution model of the species E. erythropappus in the state of Minas Gerais is consistent with the occurrence data of the species. Thus, the information generated may support studies and more sustainable management, seeking the conservation, restoration and domestication of the species. In addition, the Maxent software presented a high predictive power.

KEYWORDS: potential distribution, plant species, modeling.

\section{INTRODUÇÃO}

O estado de Minas Gerais se destaca por apresentar a maior variedade de formações vegetais, consequentemente maior riqueza em relação às angiospermas, o que pode ser explicado pela grande extensão territorial e por apresentar variações nas condições climáticas, geológicas e de relevo. Mesmo assim, apesar da importância da flora e dos inúmeros estudos já realizados, ainda não se tem total conhecimento das espécies presentes nesta biodiversidade e do seu papel nos ecossistemas (CHAVES et al., 2013).

Um dos conceitos básicos da ecologia, "o nicho ecológico" é utilizado para estabelecer padrões de riqueza e distribuição de espécie e, aliados a outros fatores, como ecológico, evolutivo e histórico, interferem sobre os padrões de distribuição de espécie. O conceito de nicho ecológico é aplicado para diversos fins, como: conservação de espécies raras ou ameaçadas, identificação dos impactos das mudanças climáticas, reintrodução de espécies, identificação de áreas com potencial para espécies invasoras (FERRARO, 2017).

A distribuição de espécies de plantas é em grande parte determinada pelo espaço multidimensional do nicho ecológico que esta ocupa incluindo fatores como o clima, fertilidade do solo, distúrbios e interações bióticas. Esses fatores atuam como filtros determinando o pool regional de espécies dentro das comunidades locais (SILVA et al., 2014). Sendo assim, os mapas de distribuição potencial de espécies auxiliam no processo de identificação de prováveis locais de ocorrência de espécies, dentre as quais, destacam-se, as espécies de múltiplos usos, como a Eremanthus erythropappus (DC.) Macleish

Conhecida popularmente por candeia, esta é uma planta da família Asteraceae e pertence ao grupo ecológico das ecótonas, sendo considerada precursora na invasão de campos. Esta espécie, por se tratar de uma heliófila, desenvolve-se em condições de plena exposição solar, como em campos abertos, formando povoamentos relativamente puros, quanto em clareiras no meio da mata. Existem várias espécies de candeia, porém a $E$. erythropappus é economicamente a de maior ocorrência em Minas Gerais (SCOLFORO et al., 2012). A madeira da candeia é muito valorizada, podendo ser empregada em uma variedade de funções devido a sua grande durabilidade. O fuste tanto pode ser utilizado como moirão de cercas quanto para extração de óleo essencial devido ao alto teor de alfabisabolol, cujo princípio ativo é amplamente utilizado na indústria farmacêutica e de cosméticos (SANTOS et al., 2015).

Neste sentido, este trabalho teve por objetivo gerar modelos da distribuição potencial atual de Eremanthus erythropappus no estado de Minas Gerais, a fim de fornecer informações que servirão como estratégia para o uso sustentável, conservação, restauração e domesticação. 


\section{Área de estudo}

\section{MATERIAL E MÉTODOS}

A área de estudo abrange todo o estado de Minas Gerais, compreendendo uma área de $586.521,235 \mathrm{~km}^{2}$, segundo o IBGE. Essa região possui áreas com relevo suave, moderado e muito acidentado com altitudes que podem variar de 500 a 2.422 metros acima do nível do mar. Os dados de ocorrência da espécie foram fornecidos pela rede species Link 2016 (Figura 1).

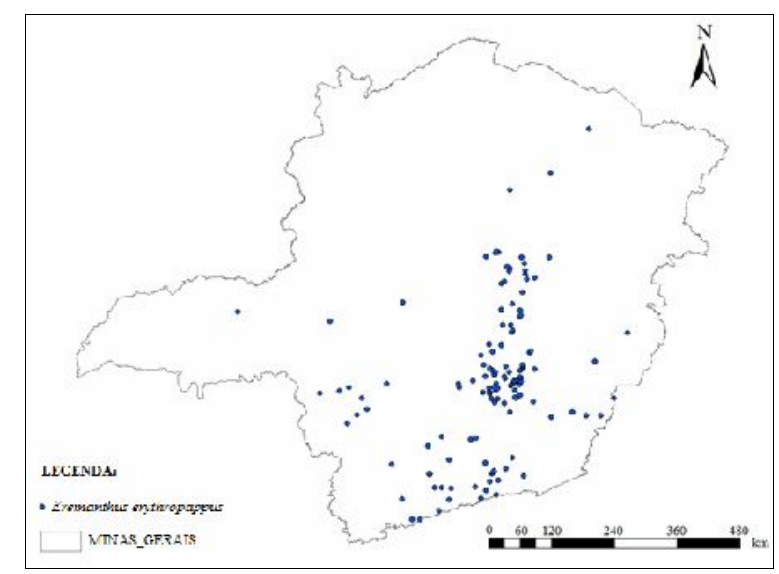

FIGURA 1. Área onde foi localizada a presença da espécie Eremanthus erythropappus no estado de Minas Gerais, Brasil. Fonte: SpeciesLink (2016).

\section{Espécie modelada}

A candeia (Eremanthus erythropappus) pertence à família Asteraceae, grupo ecológico das pioneiras sendo considerada precursora na invasão de campos abertos. A espécie apresenta baixa exigência nutricional, desenvolvendo em solos com baixa fertilidade natural e em altitude que varia entre 900 e $1800 \mathrm{~m}$. Dessa forma, a candeia se desenvolve em locais onde seria difícil a implantação de culturas agrícolas ou mesmo a implantação de alguma outra espécie florestal (AMARAL et al., 2014).

A madeira é branca ou acinzentada com grã mais escura, peso específico de $0,912 \mathrm{~g} / \mathrm{cm}^{3}$, possui casca grossa com fendas no fuste e dupla coloração nas folhas. É uma espécie florestal de múltiplos usos, sendo utilizada como moirão de cerca devido à grande durabilidade e na fabricação de medicamentos e cosméticos cujo princípio ativo é o alfabisabolol, que apresenta propriedades antibacterianas, antimicóticas, dermatológicas, antiflogísticas e espasmódicas (SANTOS et al., 2015).

\section{Dados de ocorrência}

Os registros de ocorrência de espécies foram extraídos do Species link (2016), com 143 registros de Eremanthus erythropappus referentes apenas para o território do estado de Minas Gerais. Estes dados são referentes as amostras devidamente identificadas por especialistas ou via herbário virtual.

\section{Variáveis ambientais}

Foram utilizadas 23 variáveis ambientais, sendo 19 bioclimáticas (extraídas do site Worldclim (2016), que disponibiliza dados climáticos), quatro variáveis 
topográficas (extraídas do modelo digital de elevação) (Quadro 1). As variáveis ambientais apresentam resolução de 30' e foram extraídas para o território de Minas Gerais por meio do software ArcGis 10.2.2 (ESRI, 2014).

QUADRO 1. Relação das 23 variáveis ambientais utilizadas na distribuição potencial da candeia no estado de Minas Gerais e suas respectivas descrições.

\begin{tabular}{lll}
\hline Variável & Descrição \\
\hline Bio $01 \quad$ Temperatura média anual &
\end{tabular}

Bio 02 Variação Diurna Média de Temperatura (Média mensal (Tmax-Tmin))

Bio 03 Isotermalidade ( (bio2/bio7) ( ${ }^{*}$ 100))

Bio 04 Sazonalidade da Temperatura (desvio padrão * 100)

Bio 05 Temperatura máxima do mês mais quente

Bio 06 Temperatura mínima do mês mais frio

Bio 07 Amplitude térmica anual (bio5-bio6)

Bio 08 Temperatura média do trimestre mais úmido

Bio 09 Temperatura média do trimestre mais seco

Bio 10 Temperatura média do trimestre mais quente

Bio 11 Temperatura média do trimestre mais frio

Bio 12 Precipitação Anual

Bio 13 Precipitação do mês mais chuvoso

Bio 14 Precipitação do mês mais seco

Bio 15 Sazonalidade da Precipitação (coeficiente de variação)

Bio 16 Precipitação do trimestre mais chuvoso

Bio 17 Precipitação do trimestre mais seco

Bio 18 Precipitação do trimestre mais quente

Bio 19 Precipitação do trimestre mais frio

Bio 20 Altitude STRM

Bio 21 Declividade

Bio 22 Hilshade

Bio 23 Aspect

Foram eliminadas do modelo, por meio de uma matriz de correlação ( $r>9)$, as variáveis fortemente inter-relacionadas e as de menor relevância, restando seis das 23 variáveis ambientais. Este procedimento foi realizado para evitar ou minimizar a 
overparametrization de variáveis redundantes. Assim, as variáveis ambientais mantidas foram: isotermalidade (Bio 3), sazonalidade da temperatura (Bio 4), amplitude térmica anual (Bio 7), precipitação anual (Bio 12), precipitação do trimestre mais seco (Bio 17) e altitude STRM (Bio 20) (Quadro 1). Ao final desse processo, foi obtido um mapa regional mostrando a distribuição geográfica potencial da espécie modelada.

\section{Modelagem}

Para gerar o modelo de distribuição de espécie utilizou-se o programa Maximun Entropy Species Distribution Modelling (Maxent) na versão 3.3.3, desenvolvido por Phillips et al. (2006). O algoritmo Maxent estima a probabilidade de ocorrência da espécie por meio da distribuição de probabilidade da máxima entropia e apresenta a probabilidade de ocorrência em uma escala contínua de 0 a 1 , permitindo analisar as diferenças entre as áreas de distribuição da espécie (METZ, 1986).

O mesmo algoritmo foi testado e comparado com outros modelos onde se concluiu que os resultados são mais robustos, devido ao maior acerto nas predições e destaca-se ainda o fato de necessitar apenas de dados de presença para modelar toda a área de estudo, juntamente com as informações ambientais (PHILIPS et al., 2006).

O cálculo do índice AUC e o teste estatístico Jackknife foram calculados pelas ferramentas de pós-análise disponíveis no programa Maxent. Este índice é amplamente utilizado para estimar a precisão preditiva de modelos distributivos derivados a partir de dados de presença de espécies. Ou seja, mede a capacidade de um modelo em selecionar locais de ocorrência de uma espécie versus aqueles onde a mesma encontra-se ausente (BUNGER et al. 2016; BUENO et al. 2017).

\section{RESULTADOS E DISCUSSÃO}

As variáveis selecionadas para serem utilizadas foram a isotermalidade (Bio 3), a sazonalidade da temperatura (Bio 4), a amplitude térmica anual (Bio 7), a precipitação anual (Bio 12), a precipitação do trimestre mais seco (Bio 17) e a altitude STRM (Bio 20), como apresentado na tabela 1. A Bio 20, referente a altitude STRM, é a que mais diminui o ganho quando é omitida. Isto parece, portanto, que essa possui a maioria das informações presentes em relação às outras variáveis, sendo relevante para explicar a distribuição das espécies.

TABELA 1. Resultado das estimativas de contribuição relativas das variáveis utilizadas na modelagem de distribuição da espécie Eremanthus erythropappus pelo software MaxEnt para o período atual.

\begin{tabular}{lcc}
\hline \multicolumn{1}{r}{ Variável } & Contribuição $(\%)$ & Importância de permutação \\
\hline Bio 20 & 32,8 & 30,0 \\
Bio 3 & 27,8 & 14,5 \\
Bio 12 & 17,3 & 2,4 \\
Bio 4 & 14,2 & 30,6 \\
Bio 17 & 6,7 & 17,5 \\
Bio 7 & 1,2 & 5,0 \\
\hline
\end{tabular}

O resultado do teste de Jackknife de importância para o período atual demonstrou que a variável bioclimática com maior ganho, quando usada isoladamente, foi a Bio 20. 


\section{Validação do Modelo}

A qualidade do modelo para a distribuição potencial da Eremanthus erythropappus, de acordo com o índice de acurácia AUC $(0,898)$, com um desvio padrão de 0,019, para o território de Minas Gerais foi compatível com os registros de ocorrência da espécie e a distribuição obtida, indicando que o modelo mostrou um bom desempenho na distinção de ocorrência de espécie relacionada com variáveis ambientais (METZ, 1986). O período analisado demonstrou um bom desempenho do modelo, uma vez que os valores de AUC indicam a qualidade do modelo e podem ser classificados como excelente $(1,0-0,9)$, bom $(0,9-0,8)$, médio $(0,8-0,7)$, ruim $(0,7-0,6)$ e muito ruim $(0,6-0,5)$ (METS, 1986). Valores de AUC mais próximos de 1 representa os locais de probabilidade de máxima entropia e são as áreas com nicho ecológico mais provável para a ocorrência da espécie de acordo com as variáveis utilizadas no modelo (PHILLIPS et al., 2006).

Coelho et al. (2016), utilizando o Maxent e Garp para modelagem de distribuição potencial de espécies pioneiras no estado de Minas Gerais, afirmaram que o Maxent apresentou melhores resultados que o GARP devido aos valores de AUC que foram maiores do que do algoritmo genético. Assim indicou se o uso do Maxent para buscar áreas com alta similaridade ambiental com áreas onde ocorre a espécie de interesse. O que pode diminuir a chance de erro na tentativa de introduzir a espécie no ambiente ou buscar novas populações.

Portanto, a avaliação pelo AUC foi satisfatória, visto que o desempenho do modelo pela AUC pode ser útil quando se comparam modelos de uma única espécie em um domínio geográfico semelhante (MOURA et al., 2017).

\section{Mapas de predição das espécies}

A partir dos resultados da modelagem projetou-se a ocorrência da espécie Eremanthus erythropappus no estado de Minas Gerais para o período atual (Figura 2). Quanto mais alta a ocorrência, maior a adequabilidade ambiental da espécie. A espécie Eremanthus erythropappus apresentou áreas amplas de adequabilidade ambiental no território de Minas Gerais, porém restrita à Serra do Espinhaço. A E. erythropappus possui uma ampla ocorrência no estado de Minas Gerais, podendo ser encontrada também nos Estados da Bahia, Espírito Santo, Goiás, Paraná, Rio Grande do Sul, São Paulo, Distrito Federal e Rio de Janeiro (GOMIDE et al. 2012).

Moura et al., (2017), trabalhando com a modelagem de nicho ecológico e conservação de Richeria grandis verificaram variáveis ambientais mais limitantes e ambientes com condições propícias para ocorrência da espécie, determinando portanto os fatores climáticos importantes para a ocorrência, bem como seus limites de tolerância. Além disso, a modelagem preditiva apresentou maior intensidade de adequação na mesma região onde se localizam os dados de ocorrência da espécie.

Utilizando também a ferramenta de modelagem da distribuição geográfica, Pontes e Alves (2011) buscando tratar da distribuição geográfica das espécies de Araceae ocorrentes em fragmentos de Mata Atlântica ao norte de Pernambuco, relacionando sua ocorrência aos seus respectivos hábitos e condições macroambientais, verificaram eficácia no uso da modelagem, uma vez que observaram o predomínio de espécies com distribuição disjunta sobre as de distribuição contínua e que o hábito hemiepifítico predominou em regiões úmidas e quentes, enquanto as geófitas ocorreram ainda em climas secos e/ou frios, e ainda que os "brejos de altitudes" nordestinos demonstraram ser áreas importantes para estudos biogeográficos com a família. 
Souza (2011), considerando a importância do conhecimento sobre a distribuição de espécies vegetais em matas ciliares da Chapada Diamantina e a importância de modelos preditivos no estudo de questões ecológicas e biogeográficas, realizou a modelagem da distribuição geográfica potencial de 10 espécies vegetais arbóreas indicadoras de matas ciliares desta região, no estado da Bahia, e verificando que os resultados encontrados reforçam a importância dos modelos preditivos no estudo da distribuição de espécies, sugerindo que a modelagem de nicho ecológico pode fornecer importantes contribuições para a análise de padrões biogeográficos relacionados à distribuição da flora relictual de matas ciliares, ressaltando que modelos apresentados no estudo possam subsidiar futuras pesquisas ecológicas e fitogeográficas no estado da Bahia, contribuindo no planejamento de ações e estratégias para a conservação da flora regional. Os pontos de presença foram sobrepostos às áreas de ocorrência previstas para avaliar o desempenho dos modelos (Figura 2).

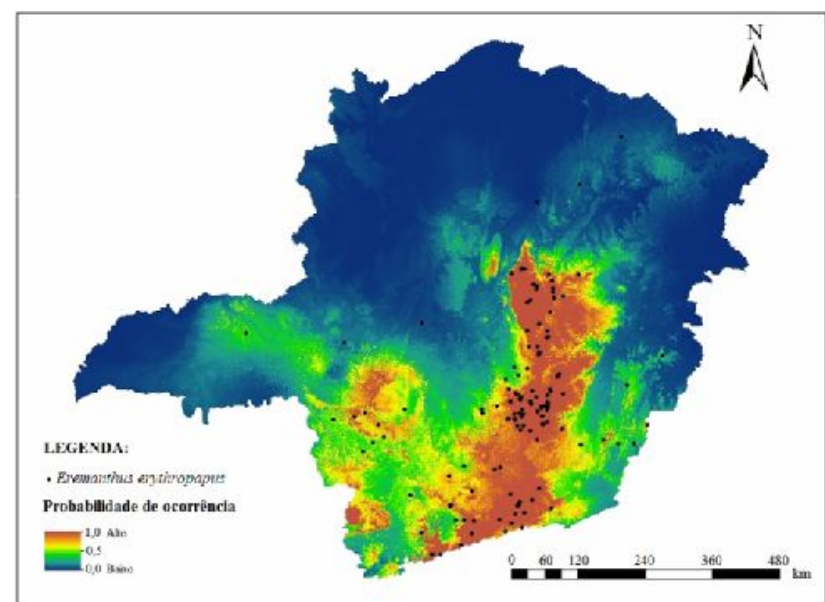

FIGURA 2. Distribuição potencial de Eremanthus erythropappus com base na adequabilidade ambiental, com os pontos de ocorrência para Minas Gerais.

\section{Avaliação dos modelos e a importância das variáveis ambientais}

Estes resultados sugerem que o programa Maxent apresentou um alto poder preditivo, considerando os dados utilizados no presente estudo. Porém contraria alguns estudos que relatam que os valores de AUC tendem a ser menores para espécies que possuem uma distribuição mais ampla (YANG et al., 2013). Devido a particularidade deste teste estatístico, os valores tendem a ser maiores para as espécies com variações ambientais mais limitadas, o que geralmente não significa que os modelos são melhores (PHILLIPS; DUDIK, 2008).

Dentre as variáveis ambientais utilizadas na modelagem da distribuição potencial deste estudo, a altitude foi a variável que mais influenciou a modelagem da candeia, sendo encontrada particularmente nos Campos de Altitude (OLIVEIRA FILHO; FLUMINHAN-FILHO, 1999). Outras variáveis que mais influenciaram o modelo foram: a amplitude térmica anual, a isotermalidade, a sazonalidade da temperatura, a precipitação anual e a precipitação do trimestre mais seco respectivamente. 


\section{CONCLUSÕES}

O uso de modelagem pode auxiliar no entendimento dos padrões de distribuição de espécies em uma análise regional, como é o caso da distribuição de Eremanthus erythropappus no Estado de Minas Gerais.

O modelo utilizado para elaborar o mapa de distribuição potencial da Eremanthus erythropappus no Estado de Minas Gerais condiz com os dados de ocorrência da espécie. Tais áreas poderão ser manejadas de forma mais sustentável, buscando a conservação, restauração e domesticação da espécie.

A modelagem de distribuição de espécies por meio do Maxent pode ser um instrumento eficaz na restauração, planejamento de conservação, assim como na recuperação de áreas degradadas.

\section{AGRADECIMENTOS}

Ao Programa de Pós-graduação em Ciência Florestal/UFVJM, pelo oferecimento da disciplina Modelagem de distribuição de espécies de espécies aplicada em parceria com o pesquisador Dcs. Marcelo Leandro Bueno. À Universidade Federal dos Vales do Jequitinhonha e Mucuri (UFVJM) e a Coordenação de Aperfeiçoamento de Pessoal de Nível Superior (Capes).

\section{REFERÊNCIAS}

AMARAL, C.S.; SILVA, E. B.; PEREIRA, I.M.; NARDIS, B.O.; GONÇALVES, N.H.; AMARAL, W.G. Crescimento da candeia pela adubação mineral e orgânica em rejeito da mineração de quartzito. Floresta, v.44, n.3, p.421-430, 2014. Disponível em < https://revistas.ufpr.br/floresta/article/view/32250/23202 >. doi: $10.5380 /$ rf.v44i3.32250

BUENO, M. L.; PONTANA, V.; NEVES, D. M.; RATTER, J. A.; OLIVEIRA-FILHO, A. T. Effects of Quaternary climatic fluctuations on the distribution of Neotropical savanna tree species. Ecography, v. 40, n. 403-414, 2017. Disponível em: < https://doi.org/10.1111/ecog.01860>. doi: 10.1111/ecog.01860

BUNGER, M. O.; MAZINE, F. F.; FOREST, F.; BUENO, M. L.; STEHMANN, J. R.; LUCAS, E. J. The evolutionary history of Eugenia sect. Phyllocalyx (Myrtaceae) corroborates historically stable areas in the southern Atlantic forests. Annals of Botany, v. 118, n. 1209-1223, 2016., Disponível em www.aob.oxfordjournals.org. doi: 10.1093/aob/mcw209

CHAVES, A. D. C. G.; SANTOS, R. M. S.; SANTOS, J. O.; FERNENDES, A. A.; MACARAJÁ, P. B. A. A importância dos levantamentos florístico e fitossociológico para a conservação e preservação das florestas. Agropecuária Científica no semiárido, v.9, n.2, p.43-48, 2013. Disponível em: <.http://dx.doi.org/10.30969/acsa.v9i2.449 . doi: 10.30969/acsa.v9i2.449

COELHO, G.L.N.; CARVALHO, L.M.T.; GOMIDE, L.R. Modelagem preditiva de distribuição de espécies pioneiras no Estado de Minas Gerais. Pesquisa Agropecuária Brasileira, v.51, n.3, p.207-214, 2016. Disponível em < http://www.scielo.br/pdf/pab/v51n3/1678-3921-pab-51-03-00207.pdf>. doi: 10.1590/S0100-204X2016000300002 
ENVIRONMENTAL SYSTEMS RESEARCH INSTITUTE (ESRI). ArcGIS Professional GIS for the desktop, versão 10.2.2, 2014.

FERRARO, J.L.S. Análise de conteúdo sobre o conceito de nicho ecológico: o que dizem os livros didáticos? Revista de Ensino de Ciências e Matemática, v.8, n.5, 35-50, 2017. Disponível em http://revistapos.cnuzeirodosul.edu.br/index.php/rencima/article/viewFile/1236/934.

GOMIDE,L.R.; ACERBI, F.W. JR.; SCOLFORO, J.R.S.; MELLO, J.M.; OLIVEIRA, A.D.; CARVALHO, L.M.T.; CALEGARIO, N.; FERRAZ-FILHO, A.C. Sustainable forest management of native vegetation remnants in Brazil. In: Martin-Garcia J, Javier Diez J, editors. Sustainable forest management: case studies . Rijeka: InTech; 2012.

METZ, C.E. ROC methodology in radiologic imaging. Investigational Radiology. v. 21, p. 720-733, sep. 1986. Disponível em: < https://www.ncbi.nlm.nih.gov/pubmed/3095258>

MOURA, C.C.; COSTA, T.R.; OLIVEIRA, P.A.; BUENO, M.L.; MACHADO, E.L.M. Modelo preditivo do potencial de distribuição geográfica da espécie Richeria grandis vahl. no estado de Minas Gerais e sua relação com as flutuações climáticas do Quaternário. Enciclopédia Biosfera, v.14, n.25, p.911- 923, 2017. Disponível em < http://www.conhecer.org.br/enciclop/2017a/agrar/modelo\%20preditivo.pdf>.doi:

10.18677/EnciBio_2017A73

OLIVEIRA-FILHO, A.T.; FLUMINHAN-FILHO, M. Ecologia da vegetação do Parque Florestal Quedas do Rio Bonito. Cerne v.5, n.2, p.51-64. 1999. Disponível em < http://www.bibliotecaflorestal.ufv.br/handle/123456789/18282>. Disponível em https://www.sciencedirect.com/science/article/pii/S0925857412003588. doi: 10.1016/j.ecoleng.2012.12.004

PHILLIPS, S. J.; ANDERSON, R. P.; SCHAPIRE, R. E. Maximum entropy modeling of species geographic distributions. Ecological Modelling, v. 190, n. 3-4, p. 231-259, 2006. Disponível em: https://www.sciencedirect.com/science/article/pii/S030438000500267X doi: 10.1016/j.ecolmodel.2005.03.026

PHILLIPS, S. J.; DUDIK, M. Modeling of species distributions with Maxent: new extensions and a comprehensive evaluation. Ecography, v. 31, p.161-175, 2008. Disponível em < https:/onlinelibrary.wiley.com/doi/10.1111/j.0906-7590.2008.5203.x>. doi: 10.1111/j.09067590.2008.5203.x

PONTES, T. A.; ALVES, M.. Padrões de distribuição geográfica das espécies de Araceae ocorrentes em fragmentos de floresta atlântica em Pemambuco, Brasil. Revista Brasileira de Biociências. Porto Alegre, v. 9, n. 4, p. 444-454, out.dez. 2011. Disponível em < http:/hww.ufrgs.br/seerbio/ojs/index.php/rbb/article/view/1845>.

SANTOS, N.O.; MARIANE, B.; LAGO, J.H.G.; SARTORELLI, P.; ROSA, W.; SOARES, M.G.; SILVA, A.M.; LORENZI, H.; VALLIM, M.A.; PASCON, R.C. Assessing the chemical composition and antimicrobial activity of essential oils from brazilian plants-Eremanthus erythropappus (Asteraceae), Plectrantuns barbatus, and $P$. 
amboinicus (Lamiaceae). Molecules, v.20, p. 8440-8452; 2015. Disponível em < file://D:/Downloads/molecules-20-08440.pdf >. doi:10.3390/molecules20058440

SCOLFORO, J. R. S.; LOEUILLE, B. F. P.; ALTOÉ, T. F. Caracterização da candeia. In: SCOLFORO, J. R. S.; OLIVEIRA, A. D.; DAVIDE, A. C. O manejo sustentável da candeia: o caminhar de uma nova experiência florestal em Minas Gerais. Lavras: Editora UFLA, 2012. p. $19-27$. SILVA, D. P. et al. Using ecological niche models and niche analyses to understand speciation pattems: The Case of Sister Neotropical Orchid Bees. PLoS ONE, v. 9, n. 11, p. 1-17, 2014. Disponível em: $<$ <htips:/joumals.plos.org/plosone/article?id=10.1371/journal.pone.0113246>. 10.1371/joumal.pone.0113246

SOUZA, H.J. Modelando a distribuição geográfica potencial de espécies vegetais arbóreas indicadoras de matas ciliares da chapada diamantina, Bahia, Brasil. Candombá - Revista Virtual, v. 7, n. 1, p. 65-75, jan-dez 2011. Disponível em: < http://revistas.unijorge.edu.br/candomba/2011v7n1/pdf/7HenriqueJesusdeSouza201 $1 \mathrm{v} 7 \mathrm{n} 1 . \mathrm{pdf}>$.

SPECIES LINK. 2016. Disponível em: < http://http://www.splink.org.br/>. Acesso em: 25 jun 2018.

YANG, X. Q.; KUSHWAHA, S. P. S.; SARAN, S.; XU, J. C.; ROY, P. S. Maxent modeling for predicting the potential distribution of medicinal plant, Justicia adhatoda L. in Lesser Himalayan foothills. Ecological Engineering, v. 51, p.83-87, 2013. Disponível em < https:/www.sciencedirect.com/science/article/pi/S0925857412003588>. doi: 10.1016/.ecoleng.2012.12.004 